УДК 004:519.713

DOI: $10.24045 /$ pp.2017.4.6

\title{
GARTHER 2017 ТОП ТЕХНОЛОГИИ: ИХ АНАЛИЗ И ПРИМЕНЕНИЕ
}

В. И. Хаханов

А. С. Мищенко

И. В. Емельянов

М. М. Любарский

Т. И. Соклакова

В. Г. Абдулаев
Доктор технических наук, профессор ORCID 0000-0001-5312-5841, e-mail: hahanov@icloud.com, научный сотрудник, преподаватель, ORCID 0000-0001-5639-5847 e-mail: alexander.mischenko@gmail.com, научный сотрудник, e-mail: iyemelyanov@itdelight.com, соискатель, e-mail:d_ad@nure.ua аспирант, ORCID 0000-0001-8763-7749, e-mail: tetiana.soklakova@gmail.com, Харьковский национальный университет радиоэлектроники, г. Харьков, Украина, кандидат технических наук, доиент, e-mail:abdulvugar@mail.com, Азербайджанская Государственная Нефтяная Академия (АГНА), Институт Кибернетики НАНА, г. Баку, Азербайджан

\section{GARTHER'S 2017 HYPE CYCLE TECHNOLOGIES: THEIR ANALYSIS AND PRACTICE}

\section{I. Hahanov}

A. S. Mishchenko

I. V. Yemelyanov

M. M. Liubarskyi

T. I. Soklakova

V. H. Abdullayev
Doctor of technical sciences, professor, ORCID 0000-0001-5312-5841, e-mail: hahanov@icloud.com, researcher, teacher, ORCID 0000-0001-5639-5847, e-mail: alexander.mischenko@gmail.com, researcher, e-mail:iyemelyanov@itdelight.com, researcher, e-mail:d_ad@nure.ua, postgraduate student, ORCID 0000-0001-8763-7749,

e-mail: tetiana.soklakova@gmail.com, Kharkov National University of Radioelectronics, Kharkov, Ukraine Candidate of technical sciences, associate professor, e-mail: abdulvugar@mail.com, Azerbaijan State Oil Academy (ASOA), Institute of Cybernetics of ANAS, Baku, Azerbaijan 
Abstract. An analytical review of the cyber physical technologies identified in the Gartner's Hype Cycle 2017 is offered, as well as some explanations related to their application in science, education, transport, industry and government structures. Selected areas are not included in the cycle of market-friendly technologies, which relate to cyber-social monitoring and management of society. It offers an expanded description of technologies related to the smart digital world, green cities and 5G-telecommunications. Recommendations are given for using the top 10 components of the super-cycle 2017 in the business and scientific-educational process of universities. The memory-driven innovative architecture of quantum computing is presented, which is characterized by the use of photon record-read transactions on the structure of electrons in the absence of logic associated with superposition and entanglement of states. A combinational QC-processor is proposed for parallel solution of the coverage problem due to hardware implementation of superposition operations, which makes it possible several times to improve the speed of procedures when searching for the optimal solution. A quantum method for minimization Boolean functions is suggested, where the states of the input variables are represented by unitary codes, which makes it possible to significantly improve the performance due to the additional memory.

Keywords: hype cycle emerging technologies; digital world; cyber-physical computing; machine learning; smart digital state; memory-driven quantum computing; logic function minimization.

1. Введение. Компания Gartner Inc., предсказывающая глобальную технологическую кибер-моду, в 2017 году добавила восемь новых трендов в свой бренд Нуре Emerging Technologies Cycle (рис. 1): 5G, Artificial General Intelligence, Deep Learning, Deep Reinforcement Learning, Digital Twin, Edge Computing, Serverless PaaS, and Cognitive Computing [1;2].

Edge computing является технологией повышения производительности облачных сервисов путем выполнения локальных вычислений по месту мобильного пользователя. Digital Twin создает кибер образы физических процессов и явлений. Как в зеркале, если нет отображения оцифрованной компании, (университета) в киберпространстве, то ее нет в физическом пространстве. Serverless PaaS - бессерверная архитектура для организации облачных вычислительных процессов на основе платформы как услуги (Platform as a Service). Экономика облачной платформы является явно выигрышной по сравнению с серверной поддержкой деятельности компаний. Поэтому весь малый и средний бизнес в течение двух лет перейдет на облачные инфраструктуры и сервисы.

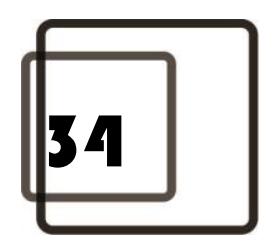




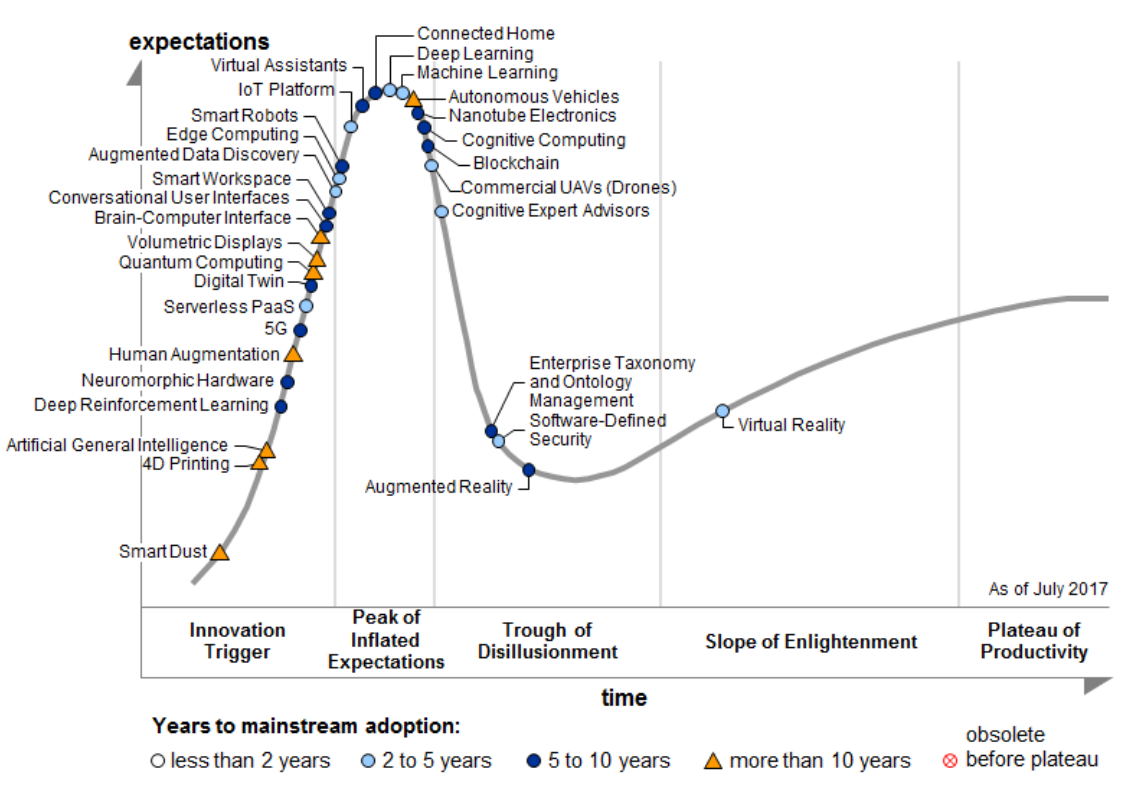

Puc. 1. Gartner's Hype Cycle for Emerging Technologies

Как следует понимать фазы Gartner цикла? 1) Innovation Trigger - запуск инновации, где потенциально интересные для рынка прорывные технологии с еще недоказанной коммерческой состоятельностью, идут на смену существующим киберфизическим укладам. 2) Peak of Inflated Expectations - пик раздутых рыночных ожиданий, где своевременная реклама создает успешные прецеденты создания инновационных технологий на фоне множества неудач. 3) Trough of Disillusionment приход разочарования, когда интерес к технологиям угасает, эксперименты не подтверждают ожидаемую рыночную привлекательность, отдельные разработчики улучшают свою продукцию и подучают инвестиции. 4) Slope of Enlightenment склон прозрения, когда появляются примеры технологий, приносящие пользу пред- приятию, находятся финансы для пилотных проектов. 5) Plateau of Productivity: плоскость устойчивого повышения производительности, когда создаваемые технологии, товары и услуги находят своего потребителя на международном рынке.

2. Три главных направления киберкультуры. Hуре-cycle 2017 формирует киберкультуру планеты на последующие 5-10+ лет путем экспертного анализа более, чем 1800 возможных технологий, выполняемого ведущими исследовательскими и консалтинговыми компаниями. Список из 33+2 топ-технологий Gartnerтаблицы создает технологическую киберкультуру, структурно представленную на рис. 2, а также конкурентные преимущества для субъектов рынка науки, образования, индустрии и транспорта. 


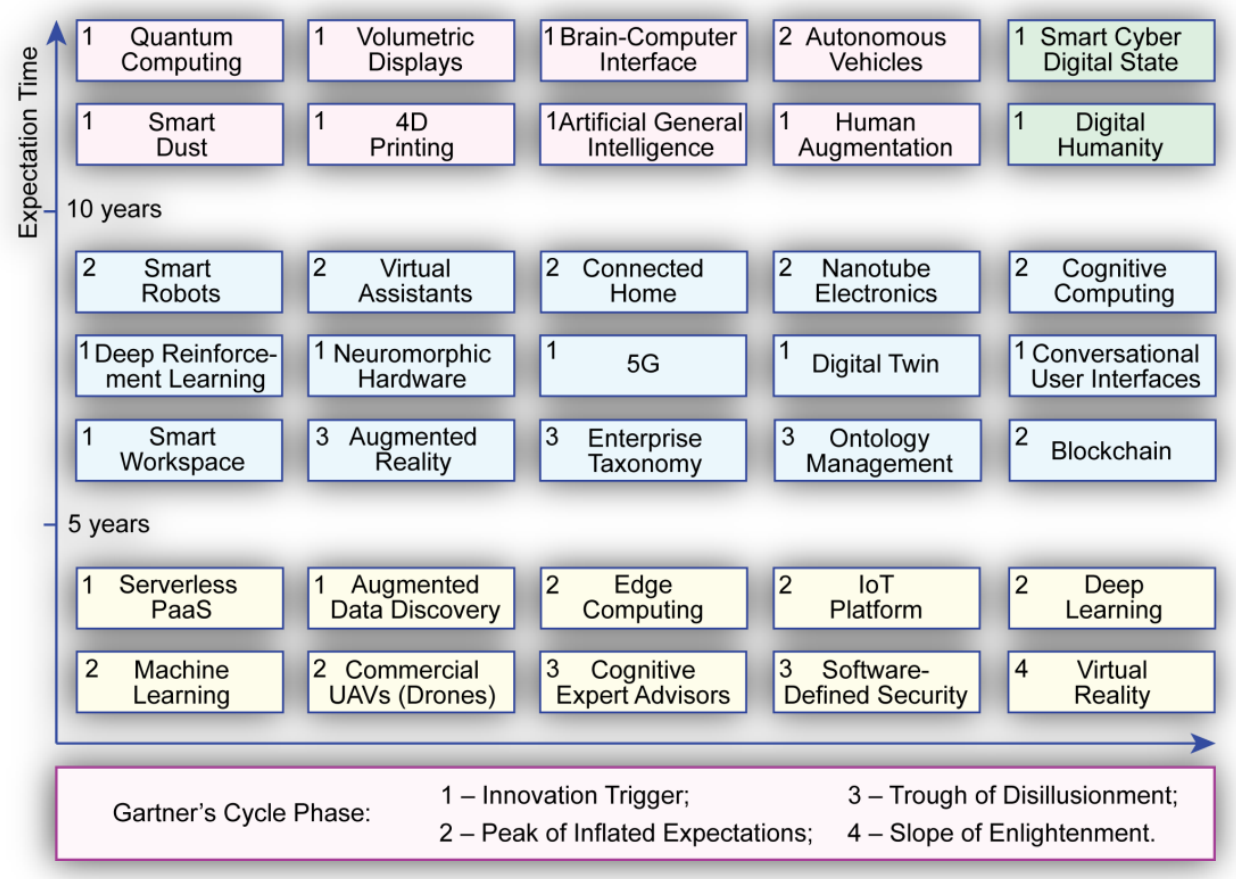

Puc. 2. Gartner's Table for Emerging Technologies

Первые три места в Gartner-топ-цикле закреплены за следующими стратегическими направленими: Artifical Intelligence Everywhere, Transparently Immersive Experiences и Digital Platforms.

1) Artifical Intelligence Everywhere. Искусственный интеллект становится самой дизрапторной технологией в последующие 10 лет благодаря наличию вычислительных мощностей, бесконечных объемов больших данных и достижений в реализации нейронных сетей для адаптации к новым ситуациям, с которыми никто и никогда не сталкивался ранее. Предприятия, которые заинтересованы в использовании искусственного

интеллекта, рассматривают полезными для себя следующие технологии: Deep Learning, Deep Reinforcement Learning, Artificial General Intelligence, Autonomous Vehicles, Cognitive Computing, Commercial UAVs
(Drones), Conversational User Interfaces, Enterprise Taxonomy and Ontology Management, Machine Learning, Smart Dust, Smart Robots and Smart Workspace. Таким образом, Artificial General Intelligence в последующие 10 лет будет проникать во все сферы человеческой деятельности, как технологическая услуга, погруженная в киберфизическое пространство, включая 30 процентов высокотехнологичных и транспортных компаний.

Smart Workspace - умное рабочее место означает быть подключенным к инфраструктуре решения производственных проблем в пространстве и времени по формату 24/7. При этом используются виртуальные частные сети, метрика измерения потенциала и результатов деятельности, наличие определенной киберкультуры и выбор наиболее удобных мест для ведения бизнеса. Высокая самомотивация к успеш- 
ному и результативному выполнению задания обусловливает использование динамически изменяющегося киберфизического рабочего пространства для творчества, инвариантного к офису, дому, транспорту, местам отдыха и спорта.

2) Transparently Immersive Experiences. Технологии опыта прозрачного погружения становятся все более ориентированными на человека и обеспечивают 1) прозрачность отношений между людьми, бизнесом и вещами; 2) гибкость и адаптивность связей между рабочим местом, домом, предприятием и другими людьми. Gartner-Inc. также предсказывает внедрение в практику следующих, ожидаемых всеми, критических технологий: Autonomous Vehicles, Brain-Computer Interfaces, Smart Dust, 4D Printing, Augmented Reality (AR), Connected Home, Human Augmentation, Nanotube Electronics, Virtual Reality (VR), and Volumetric Displays. Интеграция кибер-технологий направлена на обеспечение качества жизни человека путем создания: smart workspace, connected home, augmented reality, virtual reality and the growing brain-computer interface. Так, например, Human Augmentation технология направлена на расширение или дополнение человеческих возможностей в целях улучшения здоровья и качества жизни за счет гармоничного использования когнитивных и биотехнических улучшений, как частей человеческого тела. Volumetric Displays, как объемные дисплеи, визуализируют объекты с помощью 3D активных элементов-вокселей (voxels) в трех измерениях со сферическим углом обзора в 360 градусов, где изображение явления изменяется при перемещении зрителя. Технология 4D Printing является инновацией 3D-печати, где конструктивные материалы могут трансформироваться после производства изделия в целях адаптации продукта к потребностям человека и к окружающей среде.
3) Digital Platforms. Ключевые платформы технологической культуры формируются компонентами: 5G, Digital Twin, Edge Computing, Blockchain, IoT, Neuromorphic Hardware, Quantum Computing, Serverless PaaS и Software-Defined Security. Такие технологии, как Quantum Computing и Blockchain, будут создавать самые непредсказуемые и дизрапторные прорывы для человека в ближайшие 5-10 лет. Neuromorphic Hardware рассматривается как будущее искусственного интеллекта, которое направлено на создание нейроморфного компьютингового чипа, способного заменить облачные вычислительные мощности Apple Siri Data Center при решении сложных задач machine learning (Chris Eliasmith, a theoretical neuroscientist and co-CEO of Canadian AI startup Applied Brain Research) [3]. Иначе, внутри iphone появится цифровой мозг в форме нейроморфного IP-core, оперативно и на месте решаюший все задачи взаимодействия гаджета с внешним миром в реальном времени. Нейроморфный универсальный чип IBM, благодаря спайковому асинхронизму, потребляет на три порядка меньше энергии при количестве транзисторов, превышающих в пять раз существующие аппаратные решения компании Intel. Для программирования аппаратноориентированных алгоритмов используются компиляторы: Nengo, Python. Путем использования компилятора Nengo сегодня уже реализованы цифровые системы на кристаллах: vision systems, speech systems, motion control, adaptive robotic controllers, а также Spaun-chip для автономного интерактивного общения компьютера с окружающей средой. Software-Defined Security (SDS) или Catbird предназначена для защиты системных объектов или логических структур в виртуальном пространстве. Это связано с тем, что сетевая безопасность уже не имеет физических границ в рамках существования логиче-

Paradigmata poznání. 4. 2017 
ской архитектуры облачных сервисов. Поэтому создается точная и гибкая SDS в виде дополнения к инфраструктурам и центрам обработки данных без наличия специализированных аппаратных устройств защиты. Масштабирование SDS дает возможность создавать или приобретать минимально необходимые условия безопасности в определенном месте и времени, что существенно уменьшает материальные затраты на формирование качественного SDS сервиса.

3. Практика использования топтехнологий. Высокие затраты на исследования и разработки от Amazon, Apple, Baidu, Google, IBM, Microsoft и Facebook стимулируют создание оригинальных патентуемых решений в области Deep Learning и Machine Learning, среди которых следует отметить: Amazon Alexa, Apple Siri, Google Now, Microsoft Cortana. Koмпания Gartner Inc. уверена, что инструменты для глубокого обучения будут составлять $80 \%$ стандартных средств для ученых к 2018 году. Сегодня уже на сайтах компаний становятся доступными технологии и данные о научных исследованиях: Amazon Machine Learning, Apple Machine Learning Journal, Baidu Research, Google Research, IBM AI и Cognitive Computing, Facebook Research.

Внедрение 5G-технологии телекоммуникаций (рис. 3) в ближайшее десятилетие предоставит рынку ожидаемые инновационные решения по безопасности, масштабируемости и производительности глобальных сетей и соединений в транспорте, IoT, индустрии, здравоохранении.
Gartner Inc. прогнозирует, что к 2020 году $3 \%$ сетевых провайдеров услуг мобильной связи запустят коммерческие сети в 5G-формате, что обеспечит качественно новые условия повсеместного внедрения телекоммуникаций для масштабируемой глобализации сервисов: IoT, cloud-transport control, UHD-телевидения. Лидерами 5G-внедрения в 2017-2018 году выступают: AT\&T, NTT Docomo, Sprint USA, Telstra, T-Mobile и Verizon. Технология 5G представляет собой ультраширокополосную мобильную связь в миллиметровом диапазоне для Massive M2M транзакций в реальном времени с допустимыми для управления задержками (1мс), при одновременном подключении порядка 10 млн устройств на 1 км кв. 5G использует технологию множественного доступа с разделением луча (Beam Division Multiple Access - BDMA) для взаимодействия базовой станции с мобильными устройствами. Беспроводная сотовая архитектура $5 \mathrm{G}$ обеспечивает пропускную способность 10-50 Гбит/с в милиметровом диапазоне частот 30-300 ГГц для приложений UHD видео и создания виртуальной реальности [4]. Инновационная технология $5 \mathrm{G}$ характеризуется использованием: массива приемо-передающих антенн Massive MIMO, сети Cognitive Radio, организацией непосредственной связи D2D для IoT, созданием сети радиодоступа, как облачной услуги (radio access network as a service) и облака виртуальных сетевых функций (network function virtualization cloud - NFV).

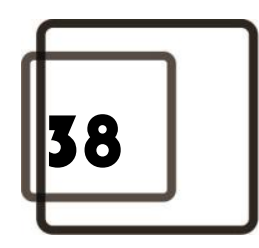




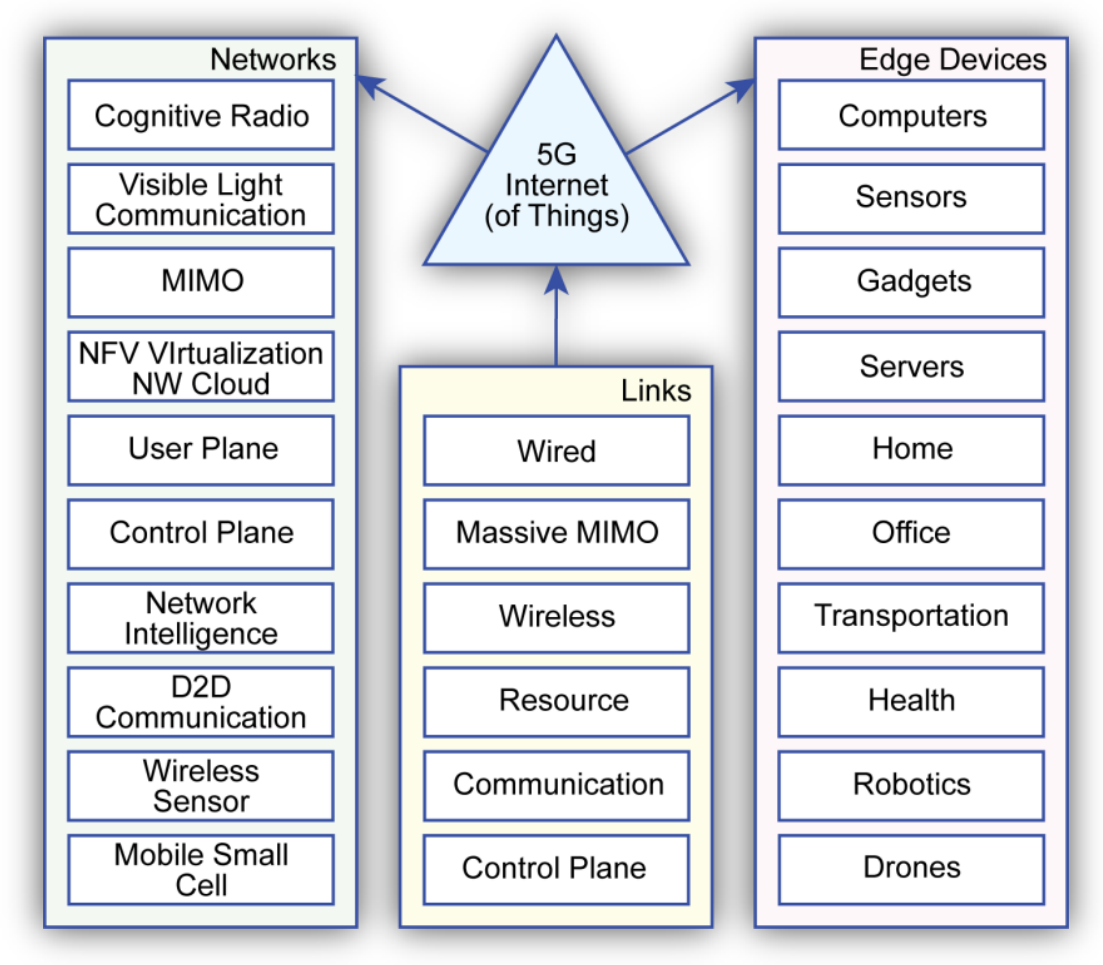

Рuc. 3. Три составляющих технологии 5G

Hype cycle, по непонятным причинам, не рассматривает две существенных для человечества абсолютно зеленые и мультимиллиардные технологии, связанные с киберсоциальным компьютингом: Digital Humanity и Smart Cyber Digital State. Они добавлены авторами публикации в Gartner's Table for Emerging Technologies, как запускаемые инновации первой фазы (innovation trigger), реализация которых ожидается через 5-10 лет.

Digital Humanity - оцифрованное человечество предполагает точную цифровую идентификацию личности по естественным биометрическим параметрам (отпечатки пальцев, сканирование лица, глаза и ДНК), исключающих бумажные носители информации, пластиковые карты, удостоверения, дипломы и паспорта в планетарном маштабе. Цифровой идентификатор дает возможность делегировать позицио- нирование каждого человека во времени и пространстве облачному сервису, который снимает все проблемы, связанные с киберфизическим анализом нелегитимных действий каждого индивидуума. Следствием устойчивого развития цифрового человечества в рамках зеленого интернета вещей для создания умного мира [5] является многомиллиардная экономия затрат на производство и использование бумажных документов, сохранение лесов и планетарной экологии. Платой за получение упомянутых дивидендов являются затраты на создание электронной инфраструктуры для цифровой аутентификации каждой личности, процесса или явления во времени и пространстве. Green IoT - киберфизическая культура человеческой деятельности, направленная на обеспечение качества жизни людей и сохранение экологии планеты, энергии, ресурсов и времени. Ком-

Paradigmata poznání. 4. 2017 
понентами IоT являются: Identification, Sensing, Controlling, Communication, Cumputation, Sevices Intelligent, Digital Infrastructure. Умный мир (smart world) предоставляет каждому человеку сервисы от: умных устройств (watches, mobile phones, computers), умного транспорта (aircrafts, cars, buses, trains), умной инфраструктуры (homes, hospitals, offices, factories, cities, states), умного образования (school, university).

Мир без посредников - это есть инновационный технологический уклад прямых телекоммуникационных контактов каждого человека с любым субъектом на планете, благодаря использованию киберфизических сервисов. Если человечество решится на уничтожение коррупции в глобальном масштабе, оно сделает это с помощью Blockchain [6]. Это есть децентрализованная криптографическая прозрачная технология хранения и обмена данными о выполненных транзакциях, которая осуществляет прямое взаимодействие бизнесменов или субъектов без доверительных юридических посредников в рамках IоTсервиса. Однако без легитимного признания Blockchain со стороны государственных структур данная технология является пока ресурсозатратным экспериментальным антикоррумпированным анклавом в киберпространстве данных и транзакций, узаконенных властью. Если уровень доверия людей к Blockchain возрастет, то может случится кибер-революция, которая низвергнет существующие государственные институты, являющиеся сегодня для налогоплательщиков затратными гарантами-посредниками в отношениях между гражданами.

Журнал IEEE Spectrum [7] опубликовал тематическую подборку статей о технологии Blockchain под девизом: "In cryptography we trust". По сути технология убирает всех "доверенных" посредников: банкиров, многомиллионную армию государственных чиновников в сделке между двумя сторонами. Третья сторона-посредник всегда является избыточной, которая уменьшает прибыль бизнесменовпроизводителей, усложняет транзакции и, что самое главное - является единственным источником коррупции на планете. «Стремление людей к секретности делает их глупыми: не видно ни плохих, ни хороших результатов. Julian Paul Assange».

Технология Blockchain (в частности, bitcoin) представляет собой киберфизический метрический облачный криптозащищенный компьютинг прозрачного мониторинга и доверительного управления транзакциями в распределенных blockchain data, puc. 4.

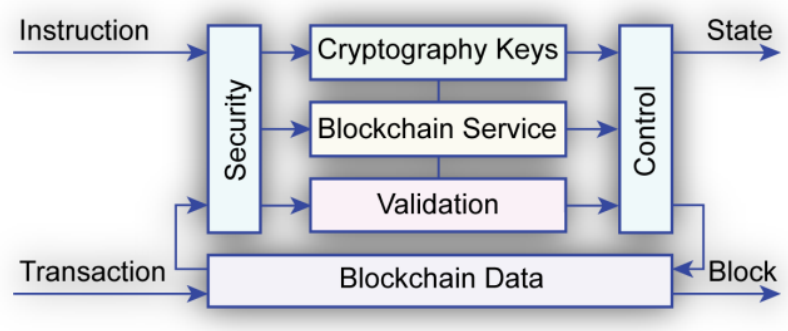

Puc. 4. Blockchain Computing

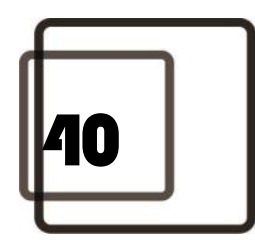


Технология, изначально предложенная Satoshi Nakamoto (2009), сегодня устойчиво развивается как распределенный в пространстве и времени доверительный компьютинг: Ethereum Virtual Machine (2013, Vitalik Buterin); Microsoft blockchain приложения на облаке Azure; IBM и Intel oткрытые pecyрсы Hyperledger. По данным Blockchain.info в проект Bitcoin сегодня уже вовлечено более 375000 человек (China, Eastern Europe, Iceland, Venezuela). Криптовалюта становится универсальным посредником между продавцом и покупателем для оценивания социальной значимости товаров и услуг. Поэтому заменить бумажные денежные знаки на виртуальные (цифровой код) будет тривиально просто, поскольку бумага не может постоять за себя. Трудности возникнут при замене миллионов чиновников, исполняющих роль посредников-доверителей, на компьютинговые облачные системы управления. Чиновники обладают властью, оружием и будут стоять насмерть для сохранения посредничества в коррумпированном в распределении денег и ресурсов, рис. 5.

Область применения технологии Blockchain - все киберфизические процессы и явления, зачастую пораженные коррупцией, благодаря наличию посредников, которые не могут быть действительно доверительными, ввиду человеческой слабости - поиметь чужое, если отсутствует наказание. Фактически можно и нужно строить Blockchain доверительные системы для открытого управления наукой, образованием, туризмом, транспортом, финансами, социумом, медициной и кадрами. Ethereum представляет собой blockchain (bitcoin) world-компьютинг, который может заменить Facebook, Twitter, Uber, Spotify, будучи неуязвимым для цензоров и прозрачным относительно происходящих процессов, а также долго работать при отсутствии людей, которые их создали.

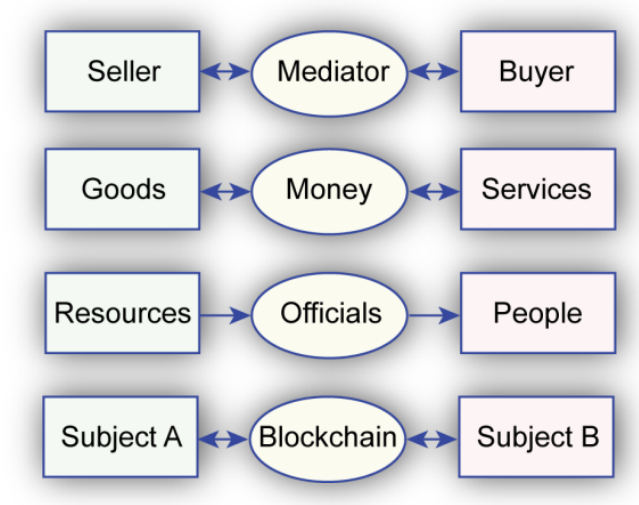

Puc. 5. Сферы применения Blockchain Computing

Недостатки Blockchain компьютинга: 1) многократное дублирование данных в распределенной сети; 2) открытость ценных данных, патентов и кодов, которая разрушит часть компаний; 3) высокие затраты на создание инфраструктуры, ориентированной на Blockchain; 4) упорное сопротивление государственных структур внедрению системы нравственного распределения ресурсов. "Money is not the root of all evil. Equity is the root of all evil", Joel Monegro. Другими словами, равенство в нищете есть причина деградации социума. 
Smart Cyber Digital State - умное кибер государство непременно состоится, как инновационная кибер социальная культура предоставления толерантных правовых, защитных и медицинских услуг на основе цифрового мониторинга и облачного управления гражданами. Почему кибергосударство должно обязательно состояться? 1) Гражданин должен иметь выбор приемлемых государственных сервисов в обмен на налоги, которые он платит в казну. Не должно существовать монополии на предоставление традиционных государственных услуг гражданину, тем более, если они логически или эмоционально становятся неприемлемыми. 2) Забота о гражданах все более превращается из территориально зависимого от места жительства в экстерриториальное облачное обслуживание, инвариантное к месту нахождения человека, благодаря наличию киберпространства, которое связывает гражданина с государственными организациями в режиме online [8]. 3) Коллизии между властью и народом должны решаться путем online мониторинга общественного мнения по существенным спорным вопросам. 4) Подобно социальным сетям, будут возникать новые кибергосударства и разрушаться старые неконкурентоспособные социальные образования. Кто будет лидером в киберпространстве, тот будет править и в физическом мире. 5) Нравственная и материальная состоятельность цели и программы развития социума, выдвигаемая политической элитой, а также ее реальных действий, становится главным аргументом рыночной привлекательности власти в борьбе за гражданина (налогоплательщика) на рынке киберфизических государств. Формула интерактивной игры при трансформировании социума проста и понятна: «Лучшим гражданам - лучшие страны». Интересно, что сегодня уже практически реализована аналогичная формула игры между вузами и учащейся молодежью в некоторых постсоветских государствах: «Лучшим студентам - лучшие университеты». 6) В киберфизическом мире постепенно меняются местами приоритеты между физическими прообразами и цифровыми образами. Пара «Master - Slave» или кто есть ведущий, а кто - ведомый, трансформируется в последовательность: «кибер образ физический прообраз». Особенно это характерно для государств, которые на протяжении всей истории человечества существовали до тех пор, пока граждане поддерживали политическую доктрину, как правило, нематериальную субстанцию, выдвигаемую властной элитой. Сегодня становится еще более важным для принятия кадровых решений не физический гражданин, а метрический кибер-образ его социальной значимости, формируемый в киберпространстве. 7) Государственность становится интегральным кибер-сервисом на рынке планеты, который может быть куплен гражданином в обмен на его налоги. 8) Главнейшей функцией кибергосударственности является формирование нравственного эмоционального интеллекma социума путем толерантного интегрирующего объединения языков, историй, культур, религий и традиций, который является доминирующим, при принятии стратегических решений, по сравнению с логическим интеллектом социальной группы. Недальновидность политической элиты, связанная с внутритерриториальным конституционным разделением граждан по метрике языков, национальностей, культур, историй и традиций неминуемо приводит к развалу государства. Политика эмоционального и логического объединения всех граждан путем толерантного отношения к упомянутым метрическим различиям создает мощный интегрирующий импульс для

Paradigmata poznńní. 4. 2017 
развития каждой личности, экономики, культуры, науки и образования.

Мир становится все более интеллектуальным, оцифрованным и сильно связанным (сетевым) по отношению к людям, вещам и услугам. На рис. 6 представлена картина, состоящая из топ-десятки стратегических тенденций в IT-индустрии 2017 года [9], которая должна быть взята на вооружение всеми компаниями и университетами, желающими формировать новые бизнес-проекты на NASDAQ-рынке товаров и услуг. Практически сегодня создается новый более совершенный умный цифровой интеллектуальный киберфизический мир совместного гармоничного существования людей в среде приятных для человека товаров и услуг. Таким образом, общая картина желаемого мира заключается в стремлении: 1) оцифровать все объекты и процессы на планете (пространственные, биологические, техниче- ские, социальные, виртуальные); 2) вдохнуть во все оцифрованные киберфизические процессы и явления масштабируемый искусственный интеллект; 3) соединить все интеллектуальные объекты и процессы в умную масштабируемую сеть в рамках оцифрованного единого киберфизического пространства. Цель создания цифрового умного интеллектуального киберфизического мира - качество жизни нравственного человека, устранение социальных пороков и зеленая экология планеты.

Компания Gartner Inc. посчитала необходимым вывести из-под зонтика актуальной рыночной моды следующие технологии, как не оправдавшие ожидания IT-бизнеса: Affective Computing, Micro Data Centers, Natural-Language Question Answering, Personal Analytics, Smart Data Discovery and Virtual Personal Assistants. 


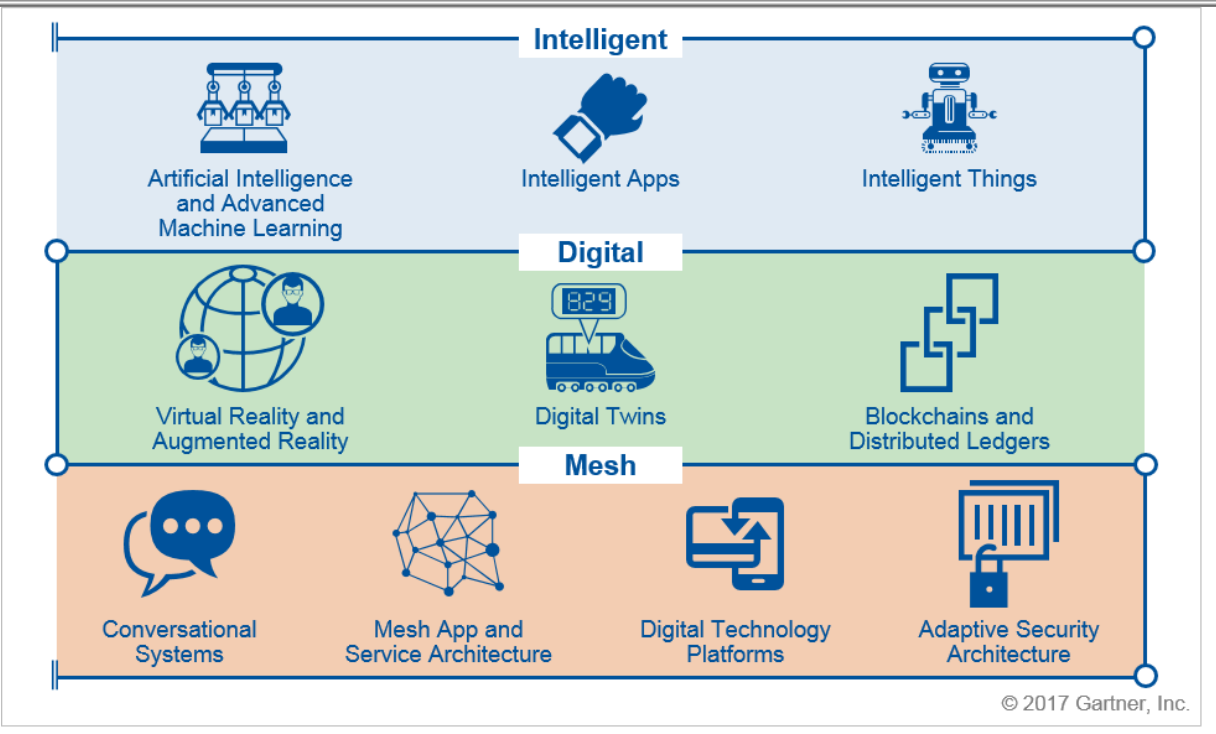

Рис. 6. Топ-10 дизрапторных направлений в IT- индустрии

Для создания успешных бизнесов и новых образовательных курсов компания Gartner Inc. рекомендует учитывать свои предположения о стратегическом планировании, которые включают включают 10 пунктов: 1) К 2020 году 100 миллионов потребителей будут делать покупки в расширенной реальности, в том числе с использованием Head-Mounted Displays (HMDs). 2) К 2020 году $30 \%$ сеансов просмотра веб-страниц будут выполняться без использования экрана. Более 5 из 550 миллионов обладателей Apple iPhone будут использовать AirPods для обмена голосовыми сообщениями. Пять процентов веб-сайтов, ориентированных на потребителя, будут оснащены аудиоинтерфейсами (включая голосовые чаты с поддержкой голоса). 3) К 2019 году $20 \%$ брендов откажутся от своих мобильных приложений (в пользу MASA - Mesh App and Service Architecture). 4) К 2020 году умные алгоритмы положительно повлияют на поведение более 1 миллиарда глобальных работников. 5) К 2022 году бизнес на основе использования блокчейнов будет стоить 10 миллиардов долларов. 6) К 2021 году $20 \%$ всех видов деятельности человека будут включены, по крайней мере, в сервисы одной из семи ведущих глобальных компаний (Google, Apple, Facebook, Amazon, Baidu, Alibaba и Tencent) 7) До 2019 года каждый доллар, инвестированный в инновации, потребует дополнительных 7 долларов для основного исполнения проекта. 8) В течение 2020 года Internet of Things (IоT) на $3 \%$ увеличит спрос, связанный с data centers. Комнатные экранные устройства, такие как Amazon Echo и Google Home, будут находиться в более, чем 10 миллионах домов. 9) К 2022 году IoT и облака (Google, Amazon, Microsoft) будут экономить потребителям и предприятиям 1 триллион долларов в год, ориентированных на услуги и расходные материалы. К 2020 году около 40 миллионов автомобилей будут использовать Android Auto, a 37 миллионов транспортных средств будут использовать CarPlay. 10) K 2020 году $40 \%$ сотрудников смогут со- 
кратить свои расходы на здравоохранение, используя фитнес-трекер.

4. Инновации для архитектуры квантового компьютинга. Физическая основа классического квантового компьютинга (рис. 7) заключается в использовании операций суперпозиции и перепутывания над состояниями электронов (p), которых вполне достаточно для организации вычислительного процесса [11-13]. Электрон выполняет функцию памяти для хранения бита информации. Транзакции между электронами осуществляются с помощью квантов или фотонов (q). Низкая и высокая орбиты электрона соответствуют значениям нуля и единицы.

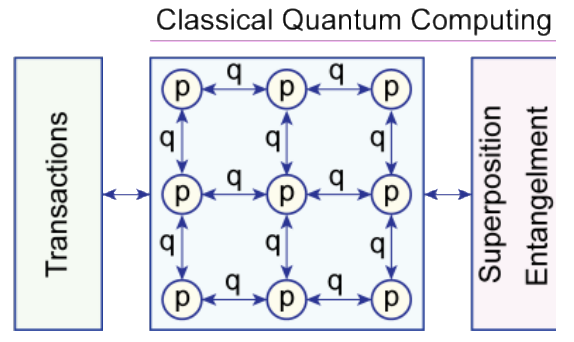

Memory-driven Quantum Computing

Рuc. 7. Два вида квантового компьютинга

Функционально полный базис для создания архитектуры квантового компьютинга представлен операциями суперпозиции и перепутывания, которым можно поставить в соответствие традиционный логический базис или-не. В теории множеств данному базису ставится в соответствие изоморфизм в форме пары “объединение-дополнение». Однозначно, операция суперпозиции в квантовой физике изоморфна логической инверсии или теоретико-множественному дополнению в алгебре логики. Поэтому естественно, что каждое состояние двоичного разряда (электрона), после применения данной операции к биту информации знает друг о друге все, где бы они не находились, с точностью до инверсии $\mathrm{a}_{\mathrm{i}} / \overline{\mathrm{a}}_{\mathrm{i}}$. Далее на основе упомянутой пары операций-примитивов (или, не) строится более сложная система логических компонентов и устройств для организации и оптимизации вычислительных процессов. Недостатки квантового классического

Paradigmala poznóní. 4. 2017 компьютинга: 1) Высокая стоимость поддержания температурных условий для функционирования квантовых атомарных структур на уровне - 270 градусов Цельсия; 2) Наблюдаемость результатов вычислительных процессов, приводящих к разрушению данных после их чтения.

Инновация в архитектуре квантового компьютинга определяется устранением логики, связанной с суперпозицией и перепутыванием. Аналогом могут выступать memory-driven архитектуры классического компьютера, свободные от reusable logic. В таком компьютере нет ничего, кроме памяти, где реализуется транзакция (операции записи-считывания данных) на адресуемой памяти. Транзакции достаточно для организации любого вычислительного процесса путем использования единственного характеристического уравнения $[11 ; 13]$ :

$$
\mathrm{M}_{\mathrm{i}}=\mathrm{Q}_{\mathrm{i}}\left[\mathrm{M}\left(\mathrm{X}_{\mathrm{i}}\right)\right] \text {. }
$$

Здесь $\mathrm{M}_{\mathrm{i}}, \mathrm{Q}_{\mathrm{i}}, \mathrm{X}_{\mathrm{i}}$ представляют собой компоненты памяти: для векторасостояния вычислительного процесса; век-

Paredigmake pornáni. 4.2017

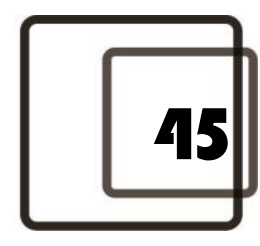


тора-кубита логического примитива; вектора-адреса ячейки логического примитива. Q-логика реализуется на адресной памяти, где также осуществляется ассемблирование всех примитивов с помощью интегрирующего М-вектора состояния вычислительного устройства, который формирует двоичные адреса $\mathrm{M}(\mathrm{X})$ на основе использования массива номеров входных переменных X.

Инновационное предложение заключается в создании квантового memory-driven компьютинга без квантовых операций суперпозиции и перепутывания (или, не) на основе использования приведенного выше характеристического уравнения, задающего две транзакции записи-считывания на структуре электронов (см. рис. 7). Исключить две сложные операции из квантового компьютинга - означает существенно упростить архитектуру и привести ее к структуре памяти на электронах для выполнения транзакций между ними с помощью квантов или фотонов.

Подтверждением состоятельности, предложенной инновационной квантовой архитектуры может служить несколько свежих публикаций, которые фиксируют устойчивую тенденцию к созданию квантового компьютинга на атомарной структуре памяти с передачей информации с помощью фотонов или квантов.

Ученые из Калифорнийского технологического института создали оптическую квантовую память [14], в которых информация передается путем кодирования данных с использованием квантового состояния фотонов. Память реализована на редкоземельных элементах и способна сохранять состояния фотонов с помощью резонаторов-посредников между атомом и светом. Размерность квантовой памяти в 1000 раз меньше, чем традиционные классические решения. Она реализована в нано по-

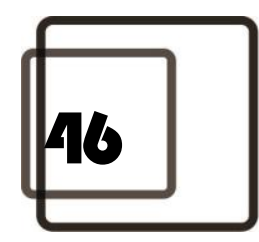

лости, которая позволяет хранить информацию в очень небольшом объеме.

Практическая реализация идеи замены электронов фотонами приводит к созданию компьютинга с быстродействием, близким к скорости света [15]. Корейские исследователи сделали еще один шаг к оптическим вычислениям. Они создали рhоton-triggered нано-проволочный транзистор на основе кристаллического и пористого кремния, где переключение и усиление величины токового сигнала осуществляется под воздействием фотона. Использовании фотонов в логических вентилях AND, OR и NAND приведет к ультракомпактным нанопроцессорам и наноразмерным фотоприемникам для получения изображений с высоким разрешением.

Ученые из Колумбийского университета провели успешные опыты по созданию транзистора из одного атома в молекулярной электронике [16]. Они реализовали геометрически упорядоченный кластер неорганических атомов с центральным ядром, состоящим из 14 атомов, которое связали с золотыми электродами, что позволило управлять транзистором под воздействием одного электрона при комнатной температуре.

Впервые достигнута передача цифровых сигналов между молекулами, что является существенным достижением на пути развития молекулярного компьютинга [17]. Создание электронных компонентов из отдельных молекул является многообещающей стратегией для миниатюризации и интеграции электронных устройств. Однако практическая реализация молекулярных устройств и схем для передачи и обработки сигналов при комнатной температуре оказалась сложной задачей, которая решена путем размещения молекул $\mathrm{SnCl}_{2} \mathrm{Pc}$ на поверхности меди $(\mathrm{Cu})$. Плоскостная ориентация молекул в межмолекулярном взаимодействии служит носителем

Paradigmata poznńní. 4. 2017 
информации. В связанных молекулярных массивах сигнал передается от одной молекулы к другой по наперед заданным маршрутам, реализующим логические операции. Явления плоскостной ориентации позволяют использовать молекулы, имеющие внутренние бистабильные состояния, для создания сложных молекулярных устройств и схем.

Теоретическое сходство классического компьютинга с квантовым заключается в общей модели вычислительной архитектуры, которая использует: память для хранения данных и функционально полный базис примитивных элементов (или, не) $=$ (суперпозиция, перепутывание) для реализации арифметико-логических операций над данными.

Каковы же формальные различия между классическим и квантовым компьютингом? Первый из них последовательно обрабатывает адресуемые или упорядоченные гетерогенные данные, затрачивая на процедуру $\mathrm{Q}=\mathrm{n}$ тактов. Он также способен обработать гомогенные данные параллельно и за один автоматный такт. Если данные не упорядочены и представляют собой множества, то предельная вычислительная сложность их обработки на классическом компьютере зависит от мощности двух множеств и определяется как $\mathrm{Q}=\mathrm{n} \times \mathrm{m}$. Например, для пересечения двух множеств: $\mathrm{M}_{1} \cap \mathrm{M}_{2}=\{\mathrm{Q}, \mathrm{E}, \mathrm{H}\} \cap\{\mathrm{E}, \mathrm{H}, \mathrm{J}\}=\{\mathrm{E}, \mathrm{H}\}$ необходимо затратить 6 автоматных тактов. Квантовый компьютинг устраняет данный недостаток, связанный с квадратичной или мультипликативной вычислительной сложностью процедуры пересечения на классическом компьютере. Он решает задачу одновременной и параллельной обработки теоретикомножественных данных. Примером тому может служить параллельное выполнение приведенной выше операции пересечения над множествами за один автоматный такт. Для этого предварительно выполняется операция суперпозиции или объединения примитивных символов, входящих B

$\mathrm{M}_{1} \cap \mathrm{M}_{2}=\{\mathrm{V}\} \cap\{\mathrm{C}\}=\{\mathrm{P}\}=\{\mathrm{E}, \mathrm{H}\}, \quad$ но при этом используется замкнутый теоретико-множественный алфавит [10]: $\mathrm{B}^{*}(\mathrm{Y})=\{\mathrm{Q}, \mathrm{E}, \mathrm{H}, \mathrm{J}, \mathrm{O}=\{\mathrm{Q}, \mathrm{H}\}, \mathrm{I}=\{\mathrm{E}, \mathrm{J}\}$, $\mathrm{A}=\{\mathrm{Q}, \mathrm{E}\}, \mathrm{B}=\{\mathrm{H}, \mathrm{J}\}, \mathrm{S}=\{\mathrm{Q}, \mathrm{J}\}, \mathrm{P}=\{\mathrm{E}, \mathrm{H}\}$, $\mathrm{C}=\{\mathrm{E}, \mathrm{H}, \mathrm{J}\}, \mathrm{F}=\{\mathrm{Q}, \mathrm{H}, \mathrm{J}\}, \mathrm{L}=\{\mathrm{Q}, \mathrm{E}, \mathrm{J}\}$, $\mathrm{V}=\{\mathrm{Q}, \mathrm{E}, \mathrm{H}\}, \mathrm{Y}=\{\mathrm{Q}, \mathrm{E}, \mathrm{H}, \mathrm{J}\}, \mathrm{U}=\varnothing\}$. Символы алфавита представляют собой множество всех подмножеств на универсуме Y, которые составлены путем суперпозиции примитивов. Квантовая суперпозиция дает возможность сосредоточить в одной точке гильбертова пространства несколько дискретных состояний. Аналогично операция объединения также создает в одной точке дискретного пространства символьный образ, содержащий несколько состояний. Исходя из сказанного, достаточно просто использовать многозначный замкнутый алфавит для моделирования квантовых вычислений на классическом компьютере. Но для этого необходимо предварительно создать символическую систему или алгебру множеств для кодирования состояний. Самой простой является алгебра Кантора, которая оперирует двумя дискретными состояниями и создает 4 символа: $\mathrm{A}^{\mathrm{k}}=\{0,1, \mathrm{X}=\{0,1\}, \varnothing\} . \quad$ Символика данного алфавита с точностью до изоморфизма представляет собой теоретикомножественную интерпретацию кубита. Иначе, суперпозиция двух состояний одного кубита образует 4 символа. Естественно, что два кубита способны сгенерировать 16 состояний, три кубита - 64 состояния. В общем случае число состояний $\mathrm{Q}$ имеет зависимость от числа куби- 
тов $\mathrm{n}$, которая представлена следующей формулой: $\mathrm{Q}=2^{2^{\mathrm{n}}}$.

Для параллельного выполнения, но уже логических операций над кубитами интерес представляет кодирование примитивных символов алфавита унарным двоичным кодом. Остальные символы по- лучаются суперпозицией кодов примитивов. Исключение составляет код символа пустого множества, который получается путем применения операции логического умножения (пересечения). Для алгебры Кантора таблица соответствия "Symbol Code" имеет следующий вид:

$$
\begin{array}{|c|cccc|}
\hline \mathrm{a}_{\mathrm{i}} \in \mathrm{A}^{\mathrm{k}} & 0 & 1 & \mathrm{X} & \varnothing \\
\hline \mathrm{C}\left(\mathrm{a}_{\mathrm{i}}\right) & 10 & 01 & 11 & 00 \\
\hline
\end{array} .
$$

Платой за параллелизм выполнения логических операций над множествами в классическом компьютере является существенное увеличение разрядности (регистра, памяти) для унарного кодирования примитивных символов алфавита. Аналогичная таблица соответствия для кодирования 16-ричного алфавита $\mathrm{B} *(\mathrm{Y})$ на четырех унарных кодах примитивов имеет вид:

\begin{tabular}{|c|cccccccccccccccc|}
\hline $\mathrm{a}_{\mathrm{i}} \in \mathrm{B}^{*}$ & $\mathrm{Q}$ & $\mathrm{E}$ & $\mathrm{H}$ & $\mathrm{J}$ & $\mathrm{O}$ & $\mathrm{I}$ & $\mathrm{A}$ & $\mathrm{B}$ & $\mathrm{S}$ & $\mathrm{P}$ & $\mathrm{C}$ & $\mathrm{F}$ & $\mathrm{L}$ & $\mathrm{V}$ & $\mathrm{Y}$ & $\varnothing$ \\
\hline $\mathrm{C}\left(\mathrm{a}_{\mathrm{i}}\right)$ & 1 & 0 & 0 & 0 & 1 & 0 & 1 & 0 & 1 & 0 & 0 & 1 & 1 & 1 & 1 & 0 \\
& 0 & 1 & 0 & 0 & 0 & 1 & 1 & 0 & 0 & 1 & 1 & 0 & 1 & 1 & 1 & 0 \\
& 0 & 0 & 1 & 0 & 1 & 0 & 0 & 1 & 0 & 1 & 1 & 1 & 0 & 1 & 1 & 0 \\
& 0 & 0 & 0 & 1 & 0 & 1 & 0 & 1 & 1 & 0 & 1 & 1 & 1 & 0 & 1 & 0 \\
\hline
\end{tabular}

Что касается табличной модели логического элемента, он изначально представлен квантоподобной совокупностью равнозначных строк или множеством дискретных отношений между входными и выходными переменными. Взамен такого множества предлагается кубитный вектор выходных состояний, ориентированный на адресное параллельное моделирование цифровых логических схем. Замена неупорядоченного множества равнозначных строк таблицы истинности на упорядоченный вектор адресуемых состояний дает возможность создавать параллельный компьютинг на классических вычислите- лях за счет увеличения объема памяти для унарного кодирования каждого состояния. Иначе, суперпозиция $\mathrm{n}$ равнозначных элементов конечного множества в квантовом (Q) компьютинге имеет взаимнооднозначное соответствие n-мерному вектору в классическом (С) адресуемом компьютинге, рис. 8. Данный вектор получен путем выполнения or-операции над унарными кодами примитивных элементов исходного множества. Естественно, что любое пересечение (and), объединение (or) или дополнение (not) в С-компьютере унарных кодов данных выполняется параллельно, за один автоматный такт, как и 
в Q-компьютере. Платой за полученное быстродействие является увеличение объема памяти (количества битов) для унарного кодирования символов относительно позиционного кодирования, которое определяется следующим выражением: $\mathrm{Q}=\mathrm{n}^{2} / \mathrm{n} \times \log \mathrm{n}=\mathrm{n} / \log \mathrm{n}$.

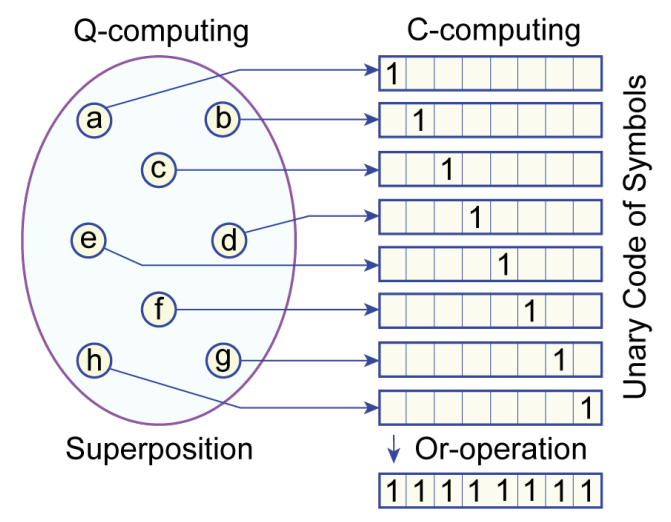

Рис. 8. Суперпозиция элементов и логическое объединение векторов

Таким образом, расширению мощности теоретико-множественного алфавита можно поставить в соответствие наращивание кубитов в квантовом компьютере. Это дает возможность параллельно и в одном автоматном такте осуществлять вычислительные процессы на основе использования логических (теоретико-множественных) операций. Суперпозиции $\mathrm{n}$ элементов конечного множества в квантовом (Q) компьютинге взаимно-однозначно соответствует n-мерный вектор в классическом (C) адресуемом компьютинге, который полу- чается на основе использования orоперации над унарными кодами символов исходного множества, выполняемой параллельно за один автоматный такт.

Интерес представляет оптимальное решение проблемы покрытия путем использования цифровой регистровой структуры (Quantum Coverage Processor QCP), создающей все возможные сочетания входных векторов в форме кубитных структур данных, представленных множеством всех подмножеств, рис. 9. 


\section{Empirical and applied research}

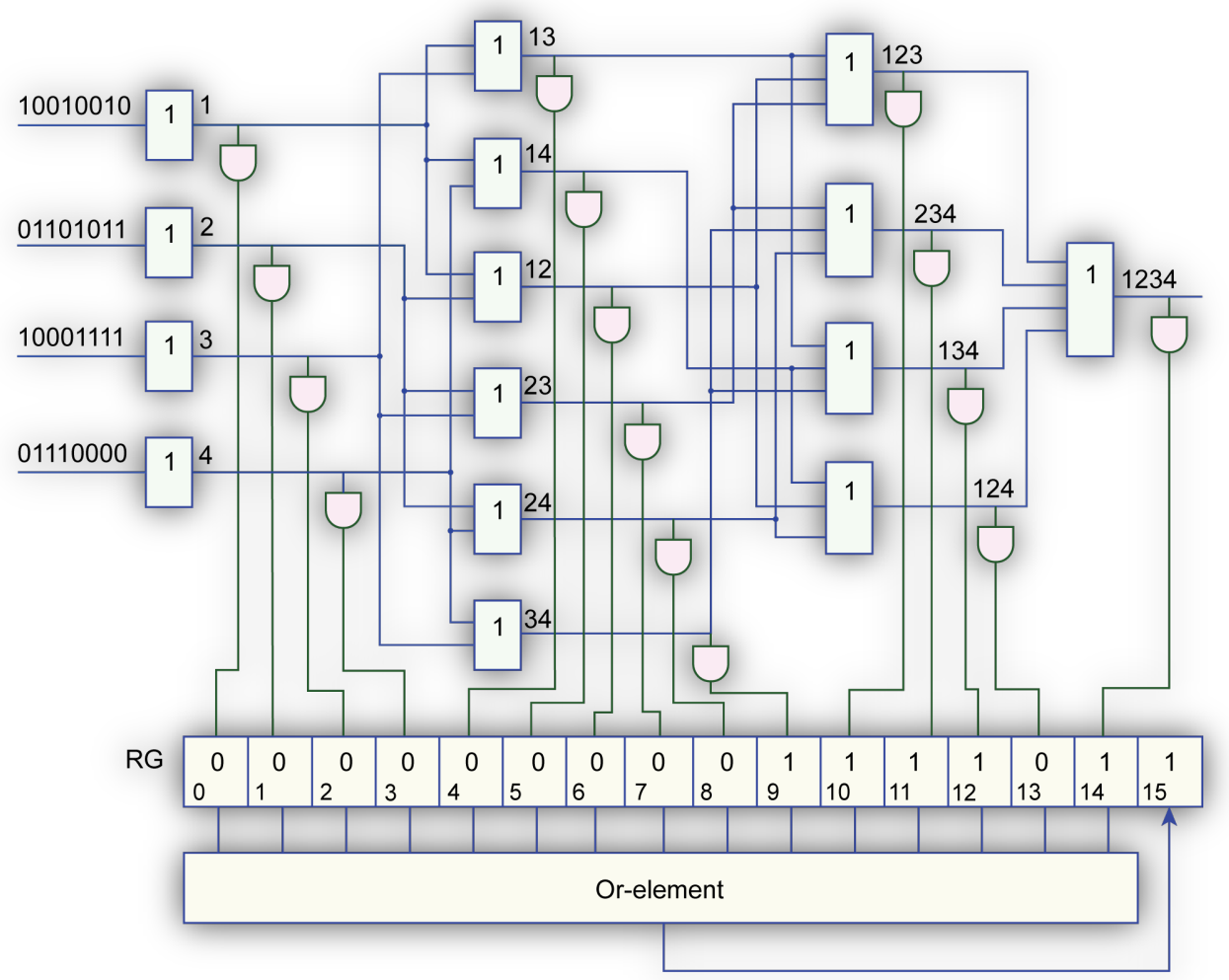

Puc. 9. QC-Processor для определения оптимального покрытия

Схема имеет логический анализатор при каждом регистровом выходе, который определяет полноту покрытия путем выполнения and-функции по всем разрядам регистровой переменной. Число таких функций-преобразователей “вектор-бит" соответствует количеству элементов и равно $\mathrm{Q}=2^{\mathrm{n}}-1$. Битовые результаты вычисления and-функций интегрируются в регистр анализа RG, который своими единичными разрядами идентифицирует получение покрытия. Последний разряд регистра, равный 1 , свидетельствует о существовании позитивного результата, полученного в процессе поиска покрытия. При этом минимальное покрытие определяется крайней левой единицей в регистре-анализаторе RG. Схема также пред- назначена для определения примитивизма или уникальности входных векторов, что идентифицируется нулевыми значениями по всем разрядам регистра-анализатора, кроме последнего, равного в этом случае единице. Аппаратная сложность регистровой цифровой схемы для поиска оптимального покрытия, где $\mathrm{n}$ - количество строк, m - длина регистра, определяется следующим аналитическим выражением: $\mathrm{Q}=\left[2^{\mathrm{n}+1}+2^{\mathrm{n}}\right] \times \mathrm{m}+2^{\mathrm{n}}$. Вычислительная сложность совокупных процедур при поиске оптимального покрытия равна, в худшем случае, n автоматным тактам. Вместо or-операций в логических элементах можно использовать хоr-функции, что позволит решать задачи по идентификации и распознаванию кибер-объектов, 
представленных в векторной форме. Здесь используется аксиома: xor-операция двух векторов-примитивов создает их логическое объединение или суперпозицию. Таким образом, $\mathrm{n}$ объектов в дискретном векторном пространстве распознаются, если все xor-сочетания, кроме последнего, формируют нулевые значения на выходе and-анализаторов.

Пример 1. Определить минимальное покрытие единичными значениями восьми разрядов следующей таблицы:

\begin{tabular}{|c|llllllll|}
\hline $\mathrm{X}$ - Inputs & 0 & 1 & 2 & 3 & 4 & 5 & 6 & 7 \\
\hline 1 & 1 & 0 & 0 & 1 & 0 & 0 & 1 & 0 \\
2 & 0 & 1 & 1 & 0 & 1 & 0 & 1 & 1 \\
3 & 1 & 0 & 0 & 0 & 1 & 1 & 1 & 1 \\
4 & 0 & 1 & 1 & 1 & 0 & 0 & 0 & 0 \\
\hline
\end{tabular}

Воздействие на схему четырьмя реги- формирует следующее состояние регистра стровыми переменными: $\mathrm{X}=(1,2,3,4)$ анализатора:

$$
\mathrm{RG}=\left[\begin{array}{lllllllllllllllll}
0 & 0 & 0 & 0 & 0 & 0 & 0 & 0 & 0 & 1 & 1 & 1 & 1 & 0 & 1 & 1
\end{array}\right.
$$

Единичные значения регистра показывают существование четырех возможных вариантов решения задачи: $\mathrm{C}=\{3,4\}$, $\{1,2,3\},\{2,3,4\},\{1,3,4\},\{1,2,3,4\}$. Минимальное покрытие обеспечивается двумя входными векторами: $\mathrm{C}=\{3,4\}$, которое идентифицируется левой крайней единицей в регистре-анализаторе RG.

QCP-структура может быть упрощена путем устранения регистра, который выполняет функцию хранения результатов поиска оптимального покрытия, рис. 10. В этом случае QCР-схема становится строго логической, где позитивный результат поиска определяется уже за один автоматный такт единичным значением состояния выхода интегрального or-элемента. Оптимальное покрытие будет идентифицироваться единичным значением выхода andэлемента, который топологически ближе находится к внешним входам схемы. 


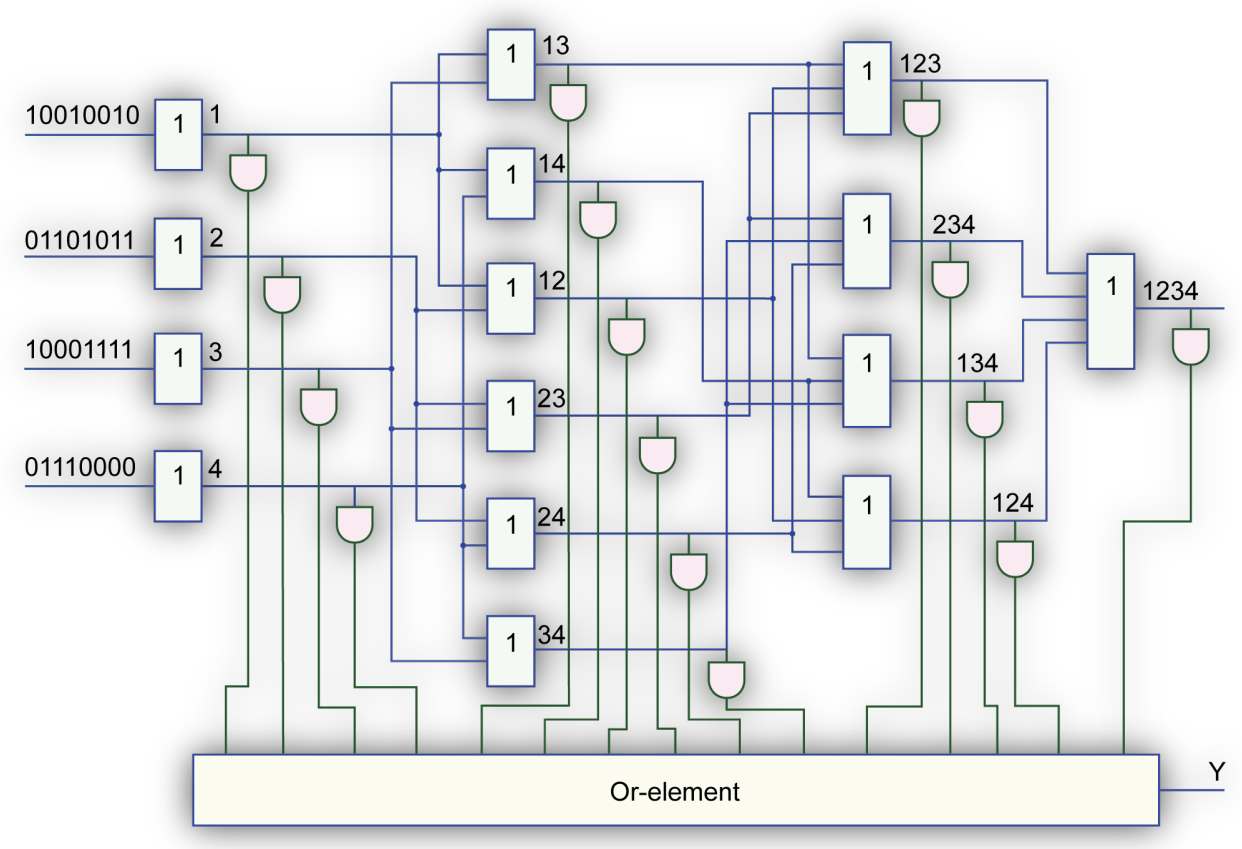

Рис. 10. Комбинационный QC-Processor для определения покрытия

Пример 2. Представлена структура из хor-элементов, которая решает задачу определения множества векторовпримитивов, подаваемых на входы циф- ровой схемы, рис. 11. Позитивный результат решения определяется наличием двух крайних правых единичных координат вектора-анализатора RG. 


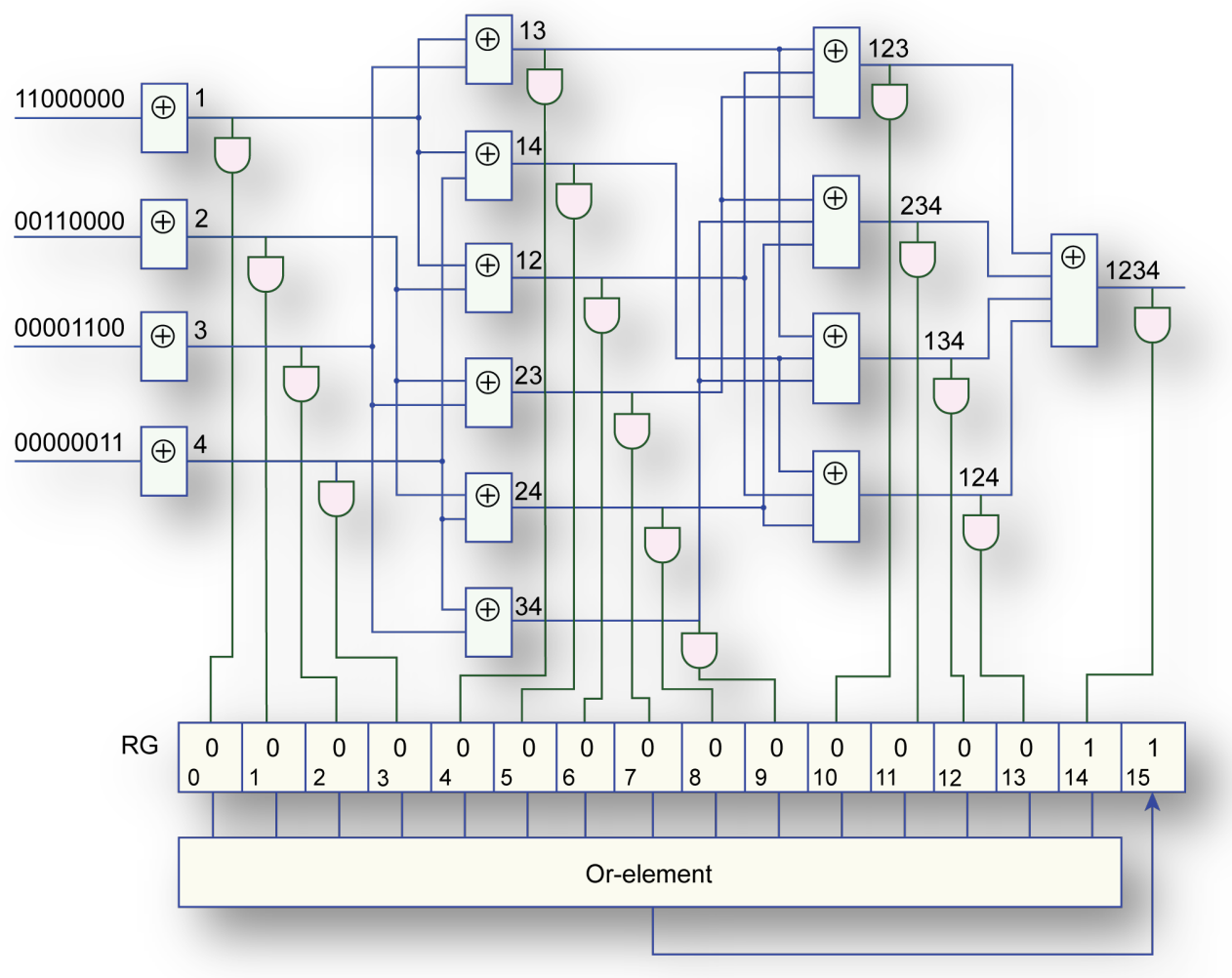

Рuc. 11. Схема определения множества векторов-примитивов

Пояснения. Хor-операция для $\mathrm{n}$ примитивов (векторов) всегда создает их объединение. Здесь речь идет о таком кодировании n объектов в дискретном киберпространстве, когда становится возможным одновременное существование или суперпозичия всех объектов в рамках формы, представленной одним вектором. Это означает, что при правильном унарном кодировании $\mathrm{n}$ объектов любая их суперпозиция, кроме последней (когда участвуют все $\mathrm{n}$ векторов), будет неполной, что приведет к появлению нулевых координат, которые сформируют значение нуля на выходах and-анализаторов.

Предложен комбинационный QCпроцессор для параллельного решения задачи покрытия, который характеризуется одновременным вычислением всех возможных комбинаторных вариантов покрытия за счет аппаратной реализации операций суперпозиции, что дает возможность в несколько раз повысить быстродействие процедур при поиске оптимального решения.

5. Метод квантовой минимизации булевых функций. Квантовое представление данных в виде суперпозиции унарных кодов можно использовать для существенного упрощения метода неопределенных коэффициентов при минимизации булевых функций.

Утверждение. Любая сколь угодно сложная таблица истинности дискретного объекта может быть представлена не более, чем двумя векторами квантового покрытия при унитарном кодировании (UC) входных состояний. Процедура, иллю-

Paradigmata poznóní. 4. 2017 
стрирующая данное утверждение представлена на рис. 12. Здесь представлены нулевые и единичные кубы таблицы истинности - входные состояния, которые унарно кодируются и логически объеди- няются (VUC). В результате получаются два вектора квантового покрытия, где каждый из которых может представлять логическую функцию в форме кубитного покрытия.

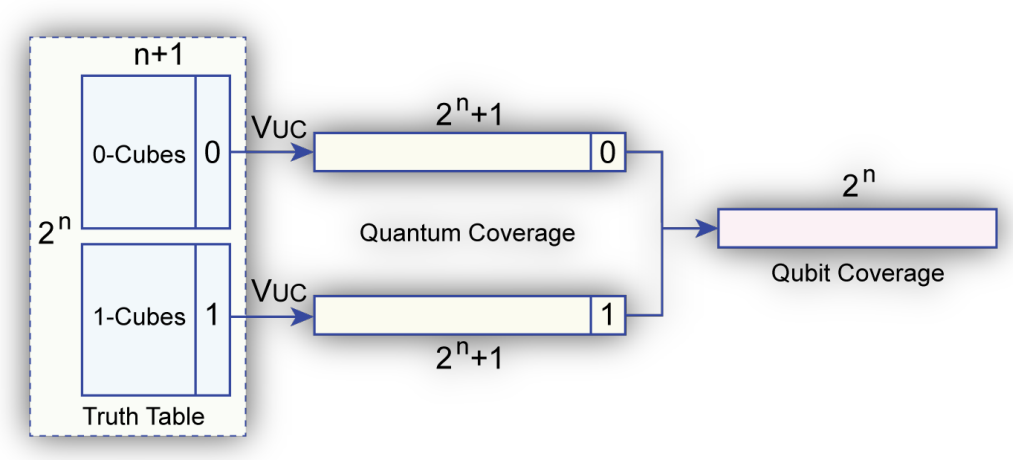

Рис. 12. Модель получения кубитного покрытия

Пусть имеется таблица $\mathrm{T}=\mathrm{T}_{\mathrm{ij}}, \mathrm{i}=\overline{1, \mathrm{~m}} ; \mathrm{j}=\overline{1, \mathrm{n}}$. В худшем случае число состояний в каждом столбце таблицы (матрицы) равно $\mathrm{m}$. Данное количество состояний может быть представлено m разрядами унитарного кода. (Например, два символа алфавита Кантора можно представить одной строкой (11), состоящей из двух двоичных разрядов:

$\left.\mathrm{A}^{\mathrm{k}}=\{0 \rightarrow 10 ; 1 \rightarrow 01 ; \mathrm{X} \rightarrow 11 ; \varnothing \rightarrow 00\}.\right)$

В результате получается матрица размерностью $\mathrm{m}$ на $\mathrm{n}$, где каждая ячейка содержит m-разрядный вектор. Применение операции суперпозиции или логического объединения ко всем строкам таблицы, благодаря унитарному кодированию, создает одну строку, которая формирует в компактном виде отношения, ранее представленные исходной таблицей. Например, все двоично-десятичные коды входных состояний логического элемента от трех переменных представляются векто- ром (1111111), если учитывать следующее кодирование: $000-10000000,001-$ 01000000, 010 - 00100000, $011-00010000$, $100-00001000,101-00000100,110-$ 00000010, 111 - 00000001. Однако, таблица истинности представляет собой функциональное соответствие

$\mathrm{Y}=\mathrm{f}(\mathrm{X}), \mathrm{X} \rightarrow \mathrm{Y}, \mathrm{X}=\left\{\mathrm{x}_{1}, \mathrm{x}_{2}, \ldots, \mathrm{x}_{\mathrm{i}}, \ldots, \mathrm{x}_{\mathrm{n}}\right\}, \mathrm{Y}=\{0,1\}$.

Учитывая, что функция определена на двух дискретных значениях $\{0,1\}$, то двоичная по выходам таблица истинности может быть всегда представлена двумя строками, каждая их которых суперпозиционирует или собирает нулевые или единичные унитарные коды входных воздействий. Например, таблица истинности xorэлемента на три входа после логического объединения унарных кодов входных состояний по единичному и нулевому значению выхода будет имет следующий вид: 


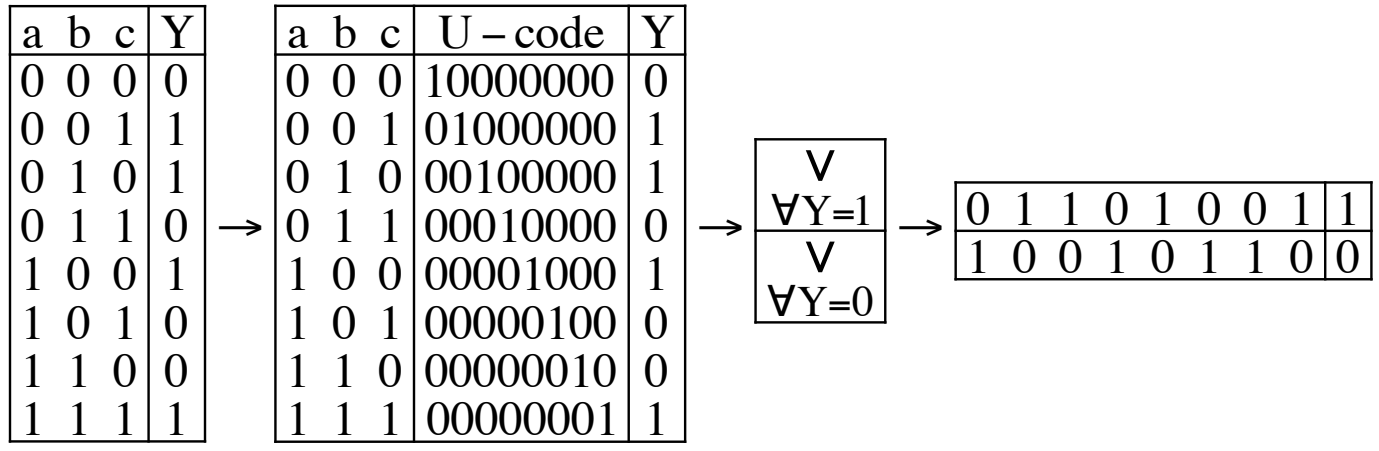

Таким образом, любая таблица истинности цифрового устройства может быть представлена в явном виде двумя строками-кубами (назовем его) квантового покрытия, которые суперпозиционно объединяют единичные и нулевые (по состоянию выхода) входные воздействия исходной таблицы. Учитывая, что кубы квантового покрытия всегда взаимно инверсны, то для задания функциональности достаточно оставить один из них, полагая, что второй можно быстро и просто до- строить с помощью операции инверсии, в случае необходимости.

С учетом использования полученной информации о возможности представления таблицы истинности двумя кубами квантового покрытия, далее предлагается модернизация известного метода неопределенных коэффициентов для минимизации логических функций. Пусть имеется исходная таблица неопределенных коэффициентов для минимизации булевой функции от трех переменных [21]:

\begin{tabular}{|c|c|c|c|c|c|c|c|c|c|}
\hline $\mathrm{T}_{\mathrm{ij}}$ & $\mathrm{x}_{1} \mathrm{x}_{2} \mathrm{x}_{3}$ & $\mathrm{k}_{1}$ & $\mathrm{k}_{2}$ & $\mathrm{k}_{3}$ & $\mathrm{k}_{12}$ & $\mathrm{k}_{13}$ & $\mathrm{k}_{23}$ & $\mathrm{k}_{123}$ & $\mathrm{f}_{\mathrm{i}}$ \\
\hline 0 & 000 & $\mathrm{k}_{1}^{0}$ & $\mathrm{k}_{2}^{0}$ & $\mathrm{k}_{3}^{0}$ & $\mathrm{k}_{12}^{00}$ & $\mathrm{k}_{13}^{00}$ & $\mathrm{k}_{23}^{00}$ & $\mathrm{k}_{123}^{000}$ & 1 \\
\hline 1 & 001 & $\mathrm{k}_{1}^{0}$ & $\mathrm{k}_{2}^{0}$ & $\mathrm{k}_{3}^{1}$ & $\mathrm{k}_{12}^{00}$ & $\mathrm{k}_{13}^{01}$ & $\mathrm{k}_{23}^{01}$ & $\mathrm{k}_{123}^{001}$ & 0 \\
\hline 2 & 010 & $\mathrm{k}_{1}^{0}$ & $\mathrm{k}_{2}^{1}$ & $\mathrm{k}_{3}^{0}$ & $\mathrm{k}_{12}^{01}$ & $\mathrm{k}_{13}^{00}$ & $\mathrm{k}_{23}^{10}$ & $\mathrm{k}_{123}^{010}$ & 1 \\
\hline 3 & 011 & $\mathrm{k}_{1}^{0}$ & $\mathrm{k}_{2}^{1}$ & $\mathrm{k}_{3}^{1}$ & $\mathrm{k}_{12}^{01}$ & $\mathrm{k}_{13}^{01}$ & $\mathrm{k}_{23}^{11}$ & $\mathrm{k}_{123}^{011}$ & 0 \\
\hline 4 & 100 & $\mathrm{k}_{1}^{0}$ & $\mathrm{k}_{2}^{0}$ & $\mathrm{k}_{3}^{0}$ & $\mathrm{k}_{12}^{10}$ & $\mathrm{k}_{13}^{10}$ & $\mathrm{k}_{23}^{00}$ & $\mathrm{k}_{123}^{100}$ & 1 \\
\hline 5 & 101 & $\mathrm{k}_{1}^{1}$ & $\mathrm{k}_{2}^{0}$ & $\mathrm{k}_{3}^{1}$ & $\mathrm{k}_{12}^{10}$ & $\mathrm{k}_{13}^{11}$ & $\mathrm{k}_{23}^{01}$ & $\mathrm{k}_{123}^{101}$ & 0 \\
\hline 6 & 110 & $\mathrm{k}_{1}^{1}$ & $\mathrm{k}_{2}^{1}$ & $\mathrm{k}_{3}^{0}$ & $\mathrm{k}_{12}^{11}$ & $\mathrm{k}_{13}^{10}$ & $\mathrm{k}_{23}^{10}$ & $\mathrm{k}_{123}^{110}$ & 0 \\
\hline 7 & 111 & $\mathrm{k}_{1}^{1}$ & $\mathrm{k}_{2}^{1}$ & $\mathrm{k}_{3}^{1}$ & $\mathrm{k}_{12}^{11}$ & $\mathrm{k}_{13}^{11}$ & $\mathrm{k}_{23}^{11}$ & $\mathrm{k}_{123}^{111}$ & 1 \\
\hline
\end{tabular}

Данная таблица преобразуется к двоичному виду всех возможных комбинаторных сочетаний состояний входных пере- менных, которые способны сформировать значения выходов функции, представленной в последнем столбце:

Paradigmata poznání. 4. 2017 


\begin{tabular}{|c|c|c|c|c|c|c|c|c|c|}
\hline $\mathrm{T}_{\mathrm{ij}}$ & $\mathrm{x}_{1} \mathrm{x}_{2} \mathrm{x}_{3}$ & 1 & 2 & 3 & 12 & 13 & 23 & 123 & $\mathrm{f}$ \\
\hline 0 & 000 & 0 & 0 & 0 & 00 & 00 & 00 & 000 & 1 \\
\hline 1 & 001 & 0 & 0 & 1 & 00 & 01 & 01 & 001 & 0 \\
\hline 2 & 010 & 0 & 1 & 0 & 01 & 00 & 10 & 010 & 1 \\
\hline 3 & 011 & 0 & 1 & 1 & 01 & 01 & 11 & 011 & 0 \\
\hline 4 & 100 & 1 & 0 & 0 & 10 & 10 & 00 & 100 & 1 \\
\hline 5 & 101 & 1 & 0 & 1 & 10 & 11 & 01 & 101 & 0 \\
\hline 6 & 110 & 1 & 1 & 0 & 11 & 10 & 10 & 110 & 0 \\
\hline 7 & 111 & 1 & 1 & 1 & 11 & 11 & 11 & 111 & 1 \\
\hline
\end{tabular}

Естественно, что полученные в ячейках таблицы комбинации входных воздействий: 0,$1 ; 00,01,10,11 ; 000,001,010$, 011, 100, 101, 110, 111 тривиально трансформируются в унитарные коды двоич- ных состояний 10,$01 ; 1000,0100,0010$, 0001; 10000000, 01000000, 00100000, 00010000, 00001000, 00000100, 00000010, 00000001 соответственно:

\begin{tabular}{|c|c|c|c|c|c|c|c|c|c|}
\hline $\mathrm{T}_{\mathrm{ij}}$ & $\mathrm{x}_{1} \mathrm{x}_{2} \mathrm{x}_{3}$ & 1 & 2 & 3 & 12 & 13 & 23 & 123 & $\mathrm{f}$ \\
\hline 0 & 000 & 10 & 10 & 10 & 1000 & 1000 & 1000 & 10000000 & 1 \\
\hline 1 & 001 & 10 & 10 & 01 & 1000 & 0100 & 0100 & 01000000 & 0 \\
\hline 2 & 010 & 10 & 01 & 10 & 0100 & 1000 & 0010 & 00100000 & 1 \\
\hline 3 & 011 & 10 & 01 & 01 & 0100 & 0100 & 0001 & 00010000 & 0 \\
\hline 4 & 100 & 01 & 10 & 10 & 0010 & 0010 & 1000 & 00001000 & 1 \\
\hline 5 & 101 & 01 & 10 & 01 & 0010 & 0001 & 0100 & 00000100 & 0 \\
\hline 6 & 110 & 01 & 01 & 10 & 0001 & 0010 & 0010 & 00000010 & 0 \\
\hline 7 & 111 & 01 & 01 & 01 & 0001 & 0001 & 0001 & 00000001 & 1 \\
\hline
\end{tabular}

Далее выполняется раздельное логическое объединение всех единичных и нулевых строк таблицы в два интегрирующих вектора. В результате получаются все возможные сочетания переменных, которые формируют единичные и нулевые значения функции. 


\begin{tabular}{|c|c|c|c|c|c|c|c|c|c|}
\hline $\mathrm{Q}$ & Operations & 1 & 2 & 3 & 12 & 13 & 23 & 123 & $f$ \\
\hline 1 & $\mathrm{Q}^{1}=\underset{\mathrm{f}_{\mathrm{i}}=1}{\mathrm{v}} \mathrm{T}_{\mathrm{ij}}$ & 11 & 11 & 11 & 1111 & 1011 & 1011 & 10101001 & 1 \\
\hline 2 & $Q^{0}=\underset{f_{i}=0}{v} T_{i j}$ & 11 & 11 & 11 & 1111 & 0111 & 0111 & 01010110 & 0 \\
\hline 3 & $Q=\left(\underset{f_{i}=1}{\vee} T_{i j}\right) \wedge\left(\overline{f_{i}=0}\left(\overline{T_{i j}}\right)\right.$ & 00 & 00 & 00 & 0000 & 1000 & 1000 & 10101001 & Y \\
\hline
\end{tabular}

Чтобы получить дизъюнктивную форму минимизированной размерности (строка 3 в приведенной выше Q-таблице), необходимо из единичного куба квантово- го покрытия логически вычесть нулевой куб по правилу, представленному в следующей формуле:

$$
\left.Q=\left(\underset{f_{i}=1}{\cup} T_{i j}\right) \backslash \underset{f_{i}=0}{\cup} T_{i j}\right)=\left(\underset{f_{i}=1}{v} T_{i j}\right) \wedge\left(\overline{f_{i}=0} T_{i j}\right) .
$$

Дешифрирование полученного квантового куба Q в дизъюнктивную нормальную форму дает следующий результат:

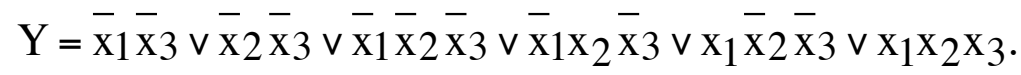

Данная форма не является минимальной, поэтому нуждается в применении средств, позволяющих решить задачу покрытия простейших исходных единичных термов $(000,010,100,111)$ полученными решениями. Для функции Ү очевидно, что первые два терма $(0 \mathrm{x} 0, \mathrm{x} 00)$ покрывают логические слагаемые $3,4,5$ или $(000,010$,
100), которые, в данном случае, являются избыточными в соответствии с правилом поглощения $\mathrm{a} v \mathrm{ab}=\mathrm{a}$, что дает возможность записать минимальную дизъюнктивную нормальную форму в следующем виде:

$$
\mathrm{Y}=\overline{\mathrm{X}} 1 \overline{\mathrm{X}}_{3} \vee \overline{\mathrm{X}}_{2} \overline{\mathrm{X}}_{3} \vee \mathrm{x}_{1} \mathrm{X}_{2} \mathrm{x}_{3} .
$$


Другое решение задачи покрытия связано с применением QC-процессора, имеющего 6 регистровых входов, что позволит определить минимальную ДНФ путем моделирования двоичных

$\mathrm{X}_{\mathrm{i}} \in \mathrm{X}$ таблицы покрытия:

\begin{tabular}{|c|cccc|c|}
\hline $\mathrm{T}$ & 000 & 010 & 100 & 111 & $\mathrm{X}_{\mathrm{i}} \in \mathrm{X}$ \\
\hline $0 \mathrm{x} 0$ & 1 & 1 &. &. & 1100 \\
$\mathrm{x} 00$ & 1 &. & 1 &. & 1010 \\
000 & 1 &. &. &. & 1000 \\
010 &. & 1 &. &. & 0100 \\
100 &. &. & 1 &. & 0010 \\
111 &. &. &. & 1 & 0001 \\
\hline
\end{tabular}

Результат моделирования кодов определяет в качестве минимального покрытия три кода-строки, которые создают минимальную функцию:

$$
\mathrm{Y}=1100 \vee 1010 \vee 0001 \rightarrow 0 \mathrm{x}_{0} \vee \mathrm{x} 00 \vee 111 \rightarrow \overline{\mathrm{x}} 1 \overline{\mathrm{x}} 3 \vee \overline{\mathrm{x}} 2 \overline{\mathrm{x}}_{3} \vee \mathrm{x}_{1} \mathrm{x}_{2} \mathrm{x}_{3}
$$

Данный метод целесообразно использовать для получения минимальной ДНФ или КНФ по таблицам истинности, где количество нулевых и единичных кубовстрок не сильно отличается друг от друга. Другое применение метода связано с существенной минимизацией дефектной области при поиске неисправностей в цифровых системах.
Вычислительная сложность Q квантового метода неопределенных коэффициентов определяется выражением, которое формирует время для унарного кодирования состояний таблицы истинности (для сравнения $\mathrm{Q}^{\mathrm{b}}$ - есть сложность базового метода минимизации):

$$
\begin{aligned}
& \mathrm{Q}=2^{\mathrm{n}} \times 3^{\mathrm{n}} ; \\
& \mathrm{Q}^{\mathrm{b}}=2^{\mathrm{n}} \times 2^{\mathrm{n}} \times 2^{\mathrm{n}}=2^{\mathrm{n}} \times 2^{\mathrm{n}+\mathrm{n}} \\
& \mathrm{R}=\frac{\mathrm{Q}}{\mathrm{Q}^{\mathrm{b}}}=\frac{2^{\mathrm{n}} \times 3^{\mathrm{n}}}{2^{\mathrm{n}} \times 2^{\mathrm{n}+\mathrm{n}}}=\frac{3^{\mathrm{n}}}{2^{2 \mathrm{n}}} .
\end{aligned}
$$

Таким образом, вычислительная сложность Q получения компактного квантового покрытия для минимизации булевых функций существенно меньше по сравнению с базовым методом $\mathrm{Q}^{\mathrm{b}}$ неопределенных коэффициентов, использующим спе- 
циальной формы таблицу истинности. Исключив предварительную обработку таблицы истинности, которая заключается в унарном кодировании состояний, вычислительная сложность собственно метода минимизации булевых функций включает всего три векторных параллельных операции.
Затраты памяти Н для хранения структур данных формируются размерностью таблицы, необходимой для суперпозиционного получения двух векторов квантового покрытия, где ячейки таблицы представлены унарными кодами состояний:

$$
\begin{aligned}
& H=2^{n} \times 3^{n} ; \\
& H^{b}=2^{n} \times 2^{n}=2^{2 n} ; \\
& S=\frac{H}{H^{b}}=\frac{2^{n} \times 3^{n}}{2^{n} \times 2^{n}}=\frac{3^{n}}{2^{n}} .
\end{aligned}
$$

Таким образом, чтобы получить компактное квантовое покрытие необходимо использовать таблицу $\mathrm{H}$ существенно большей размерности по сравнению с исходной таблицей истинности $\mathrm{H}^{\mathrm{b}}$.

Предложен квантовый способ минимизиции булевых функций, который отличается от метода неопределенных коэффициентов параллельным выполнением операции суперпозиции над нулевыми и единичными состояниями входных переменных, представленными унитарными кодами, что дает возможность существенно повысить быстродействие за счет избыточной памяти.

9. Заключение. 1. Кибер-тенденции от Gartner Inc. предоставляют возможность лидерам корпоративной архитектуры и руководителям университетов не отставать безнадежно от цифрового бизнеса в науке, образовании и индустрии, своевременно реагировать на киберфизические угрозы, возглавлять бизнес-инновации и определять эффективную цифровую бизнес-стратегию устойчивого развития государств.

2. По факту Нуре-сусlе представляет собой глубокую 4D-аналитику, во време- ни и в пространстве, состояния современного рынка устойчивого киберфизического развития умных облачных технологий на ближайшие 10-15 лет.

3. Для университетов Нуре-цикл определяет жизненную необходимость инвестировать в знания студентов инновационные технологии, показанные в фазах цикла, в целях получения через 5-10 лет армии креативных специалистов, способных поднять государство из руин современного кибер-невежества. Иначе, Gartner цикл для университета представляет собой стратегию его киберфизического устойчивого развития во времени и пространстве. Любая стратегия, разработанная без знания темпов и направления технологических изменений, будет страдать неправильным планированием действий, разрушением бизнеса, науки и образования. Например, следует учитывать, что в 2018 году robobossы будут точно мониторить и дистанционно online управлять 3 миллионами работников в мире с целью: метрически оценивать потенциал исполнителей, раздавать задания, логистически верно маршрутизировать их успешное выполнение, инвариантное к позиционированию работника в физическом про- 
странстве, оценивать качество и производительность труда, начислять заработную плату по метрическим результатам.

4. Нуре-цикл неявно дифференцирует все топ-технологии на ведущие и ведомые (master-slave), которые по факту означают, что развитию HardWare (Physical Space) платформ в сторону компактности всегда отдается приоритет, поскольку остальной виртуальный мир (Cyber Space), стремящийся к безграничному расширению SoftWare приложений всегда будет ведомым. Взаимодействие двух миров, связанных с устойчивым развитием объемов аппаратного и программного обеспечения, формирующего киберфизическое пространство, представлено на рис. 13.

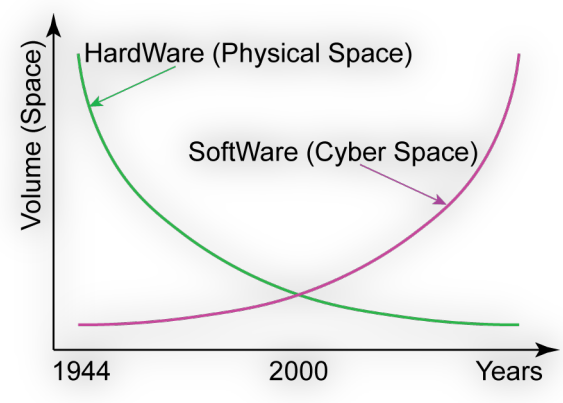

Рис. 13. Взаимодействие объемов киберфизических компонентов

5. Тем не менее, аппаратные и программные технологии представлены в Нуре-цикле (на рынке) практически в одинаковых пропорциях (50:50):

Hardware-driven technologies: 4D Printing, Volumetric Displays, Nanotube Electronics, Brain-Computer Interface, Human Augmentation, Autonomous Vehicles, Cognitive Computing, Commercial UAVs (Drones), Smart Dust, Smart Robots, Smart Workspace, Connected Home, 5G, IoT Platform, Edge Computing, Neuromorphic Hardware, Quantum Computing;

Software-driven technologies: Deep Learning, Deep Reinforcement Learning, Artificial General Intelligence, Enterprise Taxonomy, Ontology Management, Machine Learning, Virtual Assistants, Cognitive Expert Advisors, Digital Twin, Blockchain, Serverless PaaS, Software-Defined Security, Virtual Reality, Augmented Reality, Aug- mented Data Discovery, Conversational User Interfaces, Digital Humanity, Smart Cyber Digital State.

6. Одинаковое соотношение аппаратных и программных технологий в Gartnerпрогнозе означает, что уровни их капитализации на NASDAQ-рынке стремятся к паритету, ярким примером которого являются компании Apple (800 млрд долларов - индекс NASDAQ 2017) и Google (570 млрд). Эти производители существенно отличаются тем, что они полагаются на мудрость своих команд (экспертов), вооруженных доктриной: «потребители не могут предсказывать свои собственные потребности» (consumers could not predict their own needs) [9]. Альтернативой является политика компании Microsoft (503 млрд), которая проводит обширные исследования перед запуском продукта, например, такого как Windows 
Phone. По оценкам Gartner доля Apple на мировом рынке мобильных телефонов составляет $14,2 \%$ против $3,3 \%$ для Microsoft. Кому доверять, экспертам или потребителям? Ответ однозначный - экспертам, в формате 4D (всегда, везде и по всем вопросам).

7. Представлена memory-driven инновационная архитектуре квантового компьютинга, которая определяется возможностью устранения логики, связанной с суперпозицией и перепутыванием состояний на основе использования характеристического уравнения, реализующего транзакции записи-считывания на структуре электронов. Исключение логических операций из квантового компьютинга позволит существенно упростить архитектуру до уровня структуры памяти на электронах для выполнения транзакций между ними с помощью квантов или фотонов. Показано формальное отличие квантового компьютинга от классического, которое заключается в возможности параллельного и одновременного выполнения логических операций над множествами.

8. Предложен комбинационный QCпроцессор для параллельного решения задачи покрытия, который характеризуется одновременным вычислением всех возможных комбинаторных вариантов покрытия за счет аппаратной реализации операций суперпозиции, что дает возможность в несколько раз повысить быстродействие процедур при поиске оптимального решения.

9. Предложен квантовый способ минимизиции булевых функций, который отличается от известного метода неопределенных коэффициентов параллельным выполнением операции суперпозиции над нулевыми и единичными состояниями входных переменных, представленными унитарными кодами, что дает возможность существенно повысить быстродействие за счет избыточной памяти.

\section{Библиографический список}

1. https://www.forbes.com/sites/louiscolumbus/2017 /08/15/gartners-hype-cycle-for-emergingtechnologies-2017-adds-5g-and-deep-learningfor-first-time/\#646a4cf34be2

2. http://www.gartner.com/newsroom/id/3784363

3. http://www.wired.co.uk/article/ai-neuromorphicchips-brains

4. A. Gupta and R. K. Jha, "A Survey of 5G Network: Architecture and Emerging Technologies," in IEEE Access, vol. 3, pp. 1206-1232, 2015.

5. C. Zhu, V. C. M. Leung, L. Shu and E. C. H. Ngai, "Green Internet of Things for Smart World," in IEEE Access, vol. 3, pp. 2151-2162, 2015.

6. K. Christidis and M. Devetsikiotis, "Blockchains and Smart Contracts for the Internet of Things," in IEEE Access, vol. 4, pp. 2292-2303, 2016.

7. Blockchains: How They Work and Why They'll Change the World IEEE Spectrum. October 2017. https://spectrum.ieee.org/computing/networks/blo ckchains-how-they-work-and-why-theyll-changethe-world

8. A. Zanella, N. Bui, A. Castellani, L. Vangelista and M. Zorzi, "Internet of Things for Smart Cities," in IEEE IoT Journal, vol. 1, no. 1, pp. 22-32, Feb. 2014.

9. https://www.gartner.com/doc/3471559?srcId=1$7578984202 \& u t m$ campaign=RM GB 2017 TRENDS_QC_E2_What\&utm_medium $=\mathrm{e}$ 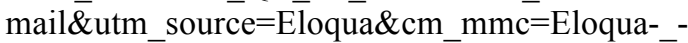 Email- -

LM_RM_GB_2017_TRENDS_QC_E2_What-_0000

10. http://www.gartner.com/smarterwithgartner/threedigital-marketing-habits-to-break-2/

11. Vladimir Hahanov. Cyber Physical Computing for IoT-driven Services. New York. Springer. 2017. - 243p.

12. Hahanov V.I., Bani Amer Tamer, Chumachenko S.V., Litvinova E.I. (2015) Qubit technology for analysis and diagnosis of digital devices. Electronic modeling. J 37 (3): 17-40.

13. Vladimir Hahanov, Wajeb Gharibi, Eugenia Litvinova, Mykhailo Liubarskyi, Anastasia Hahanova. Quantum memory-driven computing for test synthesis. IEEE East-West Design and Test Symposium. 2017. Novi Sad, Serbia. P. 123-128.

14. Tian Zhong1, Jonathan M. Kindem1, John G. Bartholomew1 at all. Nanophotonic rare-earth quantum memory with optically controlled retrieval. Science. 29 Sep 2017: Vol. 357, Issue 6358, pp. 1392-1395. 
15. Jungkil Kim, Hoo-Cheol Lee, Kyoung-Ho Kim at all. Photon-triggered nanowire transistors. Nature Nanotechnology 12, pp. 963-968 (2017).

16. Giacomo Lovat, Bonnie Choi, Daniel W. Paley at all. Room-temperature current blockade in atomically defined single-cluster junctions. Nature Nanotechnology, doi:10.1038/nnano.2017.156.

17. Chao Li, Zhongping Wang, Yan Lu, Xiaoqing Liu \& Li Wang. Conformation-based signal transfer and processing at the single-molecule level. Nature Nanotechnology.

(2017), doi:10.1038/nnano.2017. 179.

18. Patrick T. Hester, Kevin Adams. Systemic Decision Making. Fundamentals for Addressing Problems and Messes. Springer Nature. 412 p.
19. Hahanov Vladimir, Barkalov Alexander, Adamski Marian. Infrastructure intellectual property for SoC simulation and diagnosis service. Springer, Germany. 2011. - P. 289-330.

20. http://dpchas.com.ua/politika/dubilet-zadumalrevolyuciyu

21. Савельев А. Я. Прикладная теория цифровых автоматов. - М. : Высш. шк., 1987. - 272 с. (Savel'ev A. Ja. Prikladnaja teorija cifrovyh avtomatov. - M. : Vyssh. shk., 1987. - 272 c.)

(C) Хаханов В. И., Мищенко А. С., Емельянов И. В., Любарский М. М., Соклакова Т. И., Абдулаев В. Г., 2017. 